\title{
Variable Viscosity Effects on Unsteady MHD an Axisymmetric Nanofluid Flow over a Stretching Surface with Thermo-Diffusion: FEM Approach
}

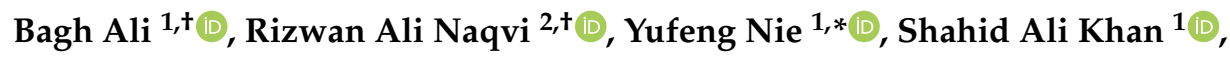 \\ Muhammad Tariq Sadiq ${ }^{3}{ }^{(0)}$, Ateeq Ur Rehman ${ }^{4}\left({ }^{-}\right.$and Sohaib Abdal ${ }^{5}$ (i) \\ 1 Department of Applied Mathematics, School of Science, Northwestern Polytechnical University, \\ Dongxiang Road, Chang'an District, Xi'an 710129, China; baghalisewag@mail.nwpu.edu.cn (B.A.); \\ shahidalimaths@mail.nwpu.edu.cn (S.A.K.) \\ 2 Department of Intelligent Mechatronics, Sejong University, Seoul 100083, Korea; rizwanali@sejong.ac.kr \\ 3 School of Automation, Northwestern Polytechnical University, 127 West Youyi Road, Xi'an 710072, China; \\ tariq.sadiq@mail.nwpu.edu.cn \\ 4 College of Internet of Things Engineering, Hohai University, Changzhou 213022, China; \\ ateqrehman@gmail.com \\ 5 School of Mathematics, Northwest University, No. 229 North Taibai Avenue, Xi'an 7100069, China; \\ sohaib@stumail.nwu.edu.cn \\ * Correspondence: yfnie@nwpu.edu.cn \\ $\dagger$ Bagh Ali and Rizwan Ali Naqvi are Co-first author, these authors contributed equally to this work.
}

Received: 24 December 2019; Accepted: 27 January 2020; Published: 4 February 2020

\begin{abstract}
The present study investigated the unsteady magnetohydrodynamic (MHD) nanofluid flow over a radially nonlinear stretching sheet along with the viscosity dependent on temperature, convective boundary condition, thermo-diffusion, and the radiation effects. Moreover, the nanofluid's viscous effects were considered dependent on temperature and the exponential Reynolds model was considered in this context. It was additionally assumed that a uniform suspension of nanoparticles is present in the base fluid. The Buongiorno model, which involves the thermophoresis and Brownian motion effects, was considered. For the sake of a solution, the variational finite element method was selected with coding in MATLAB and the numerical results were contrasted with the published articles. The influence of various physical parameters on the velocity, temperature, and concentration profiles are discussed by the aid of graphs and tables. It was detected that the nanofuid viscosity parameter declines the fluid flow velocity, while, for the temperature and the concentration profiles, it accomplished the reverse phenomenon.
\end{abstract}

Keywords: nano-fliud; themo-diffussion; MHD; finite element method; convective surface bounday conditions; variable vioscosity

\section{Introduction}

The exploration of unsteady nano-fluid flow through a nonlinear stretching surface has been fundamentally prolonged for the impressive consideration during the most recent years because of numerous appliances in the engineering field. It consists of a micro-electro-mechanical structure that is highly developed in nuclear schemes; glass fiber, fuel cells, and paper fabrication have an imperative part in our daily life. A suspension containing nanoparticles in a base liquid (water, mixture of base fluid, kerosene, biofluids, and organic liquids), which changes the base fluid's viscosity, thermal conductivity, density, and mass diffusivity, is called a nanofluid. The composition of nanoparticles is made from metal nitride, metals, oxide, ceramics, and carbide ceramics. The erudition of magneto 
hydrodynamics is a momentous chore as it has insinuations in industry as well as flow phenomena. In the last few centuries, enterprises to investigate the fluid physics at the nano and micro scales have enhanced. Aziz et al. [1] described that the change in flow geometry, enhancing thermal conditions, using porous medium and boundary conditions can improve heat transfer capacity of the fluid. The perceptions of nano-fluid were commenced by Choi [2] to illustrate that a base fluid such as water or ethylene-glycol mixture can improve its thermal conductivity with the addition of nanoparticles. Ariel [3] portrayed a model of axisymmetric flow caused by radically stretched sheet and gauged the consequences by the finite difference method. Recently, Mustafa et al. [4] deliberated that the nanofluids flow through radially stretching sheet and evaluated it both numerically and analytically. Akbari et al. [5] examined the impact of nanoparticles existing of non-Newtonian nano-fluid and illustrated that thermal conductivity enlarges due to enhancement in the nanoparticles. Mohyud-Din et al. [6] similarly scrutinized the performance of nanoparticles.

In the flow of a fluid, thermal radiation is significant and therefore the impacts of thermal radiation on heat and mass transfer have been widely studied. Raza [7] explored the effects of radiation on temperature. Ashraf and coauthors [8] also investigated the thermal conductivity impacts of the non-Newtonian fluid. Chen [9] inspected the mixed convection fluid flow over a stretching sheet. Speculating on a micro-polar fluid, Sankara et al. [10] scrutinized the micro-polar fluids flow through a stretching sheet. The consequences of a magnetic field on the constricting viscous fluid flow along with the parallel plates were conferred and explained using the perturbation method by Hamza [11].

The fluid flow of the boundary layer induced by the stretching sheet is an important form of flow occurring in flow processes in chemical industries and engineering. These incorporate paper production, fluid metal, glass fiber, and polymer sheet manufacturing. Non-Newtonian fluids, including physiological fluids, oils, paints, natural fluids, colloidal fluids, and foodstuffs, play a significant role in our daily lives [12,13]. Rana et al.[14] studied the impact of mixed convection and magnetic field on the thermal conductivity in the presence of nanoparticles. Thumma et al. [15] investigated the numerical study of heat source on dissipative magnetic nanofluid flow from a nonlinear inclined stretching surface. Seth et al. [16] analyzed the effects of radiation and chemical on the MHD natural convection heat and mass transfer flow. Takhar et al. [17] studied the Mixed convection flow of a micro-polar fluid over a stretching sheet. Qiu et al. [18] reviewed recent advances in thermophysical properties at the nanoscale from solid state to colloids. For viscous dissipation, Dhanai et al. [19] studied the MHD heat transfer flow and investigated the dual solution. Nayak et al. [20] found steady axisymmetric flow and heat transfer of fluid flow over a radially stretching surface. Ashraf et al. [21] analyzed the magnetohydrodynamic flow of non-Newtonian fluid and also studied the heat flow utilizing the stretchable disk. They acquired the numerical outcomes of an axisymmetric flow over a stretchable surface. Faraz et al. [22] studied the transfer of heat from an axisymmetric viscous fluid over a stretching radially surface.

The novelty of this work is to consider the Reynolds exponential viscosity model with convective boundary condition over radially nonlinear stretched sheet, heat and mass transfer characteristics of the thermo-diffusion, and radiation effects. Another aspect of this work is the numerical method of solution, especially the finite element method (FEM) was chosen, which is the most robust method to solve the differential equations [23,24]. Kumar et al. [25] described that finite element method is especially utilized in business software akin to MATLAB, ADINA, Abaqus, and Ansys.

The article is organized as follows. Section 1 is the introduction, which contains the literature review. In Section 2, we model the physical problem, starting from the modeled partial differential equations. Then, we transform PDEs into ODEs using appropriate similarity transformation. Section 3 is completely devoted to develop the variational finite element method solution. In Section 4, the graphic plots are presented under the influence of several important entities, as well as a detailed analysis of velocity, temperature, and concentration profiles. Further, a comparison of the skin friction coefficient and Nusselt number is made with the numerical results reported in the literature to confirm 
the validity of the present consequences of the finite element method. Finally, the concluding remarks are describe in Section 5.

\section{Mathematical Formulation}

Let us consider an unsteady magnetohydrodynamic flow of incompressible viscous flow of nano-fluid over a nonlinear stretching sheet, with $z=0$. The flow of the conducting fluid is assumed to be in the radial direction $U_{w}=c_{0} r^{m} /(1-\lambda t)$, where $c_{0}>0, m \geq 0$ are dimensional constant. The temperature of the sheet is regulated by a convective heating process characterized by hot fluid $T_{f}$ temperature and variable heat transfer coefficient $h_{f}(x)$, solutal concentration, and nanoparticle concentration represent $\left(C_{w}\right.$ and $\psi_{w}$, respectively). The ambient values of the temperature, solutal concentration, and nanoparticle concentration are denoted by $T_{\infty}, C_{\infty}$, and $\phi_{\infty}$, respectively (see Figure 1). $B(r, t)$ denotes the variable magnetic field intensity where $t$ is time. The magnetic field acts normally to the sheet in the positive z-direction and $B(r, t)$ generalizes the magnetic field term previously given in [26] to $B(r, t)=B_{0} r^{\frac{(m-1)}{2}} / \sqrt{(1-\lambda t)}$. $B_{0}$ is the uniform strength of the magnetic field, $m>0$ is the power-law index, and $\lambda$ denotes the unsteadiness parameter. Under the above conditions, the governing equations of continuity, momentum conservation, energy conservation, and nanoparticle volume fraction can be expressed as (see [12,27]):

$$
\begin{gathered}
\frac{\partial u}{\partial r}+\frac{u}{r}+\frac{\partial w}{\partial z}=0 \\
\rho\left(\frac{\partial u}{\partial t}+u \frac{\partial u}{\partial r}+w \frac{\partial u}{\partial z}\right)=\frac{\partial}{\partial z}\left(\mu(T) \frac{\partial u}{\partial z}\right)-\sigma B^{2}(r) u \\
\frac{\partial T}{\partial t}+u \frac{\partial T}{\partial r}+w \frac{\partial T}{\partial z}=\alpha\left(1+\frac{16 T_{\infty}^{3} \sigma^{*}}{3 k^{*} \kappa}\right) \frac{\partial^{2} T}{\partial z^{2}}+\tau D_{B} \frac{\partial \psi}{\partial z} \frac{\partial T}{\partial z}+\tau \frac{D_{T}}{T_{\infty}}\left(\frac{\partial T}{\partial z}\right)^{2}+D_{T C} \frac{\partial^{2} C}{\partial^{2} z} \\
\frac{\partial C}{\partial t}+u \frac{\partial C}{\partial r}+w \frac{\partial C}{\partial z}=D_{s} \frac{\partial^{2} C}{\partial z^{2}}+D_{C T} \frac{\partial^{2} C}{\partial^{2} z} \\
\frac{\partial \psi}{\partial t}+u \frac{\partial \psi}{\partial r}+w \frac{\partial \psi}{\partial z}=D_{B} \frac{\partial^{2} \psi}{\partial z^{2}}+\frac{D_{T}}{T_{\infty}} \frac{\partial^{2} T}{\partial^{2} z}
\end{gathered}
$$

where $u$ and $w$ are component of velocity along $r$ and $z$ directions, respectively; $\sigma, v$, and $\rho$ are the electrical conductivity, kinetic viscosity, and viscosity of fluid, respectively; $D_{B}$ and $D_{T}$ are the Brownian diffusion and thermophoretic diffusion coefficient, respectively; $D_{S}, D_{C T}$, and $D_{T C}$ are the solutal, Soret and Dufour diffusities, respectively; $\sigma^{*}$ is the Stefan-Boltzmann constant; $k^{*}$ is the mean absorption coefficient; and $\alpha$ is the thermal diffusivity of the fluid. The corresponding boundary conditions are (see $[20,28])$ :

$$
\begin{gathered}
u=U_{w}, w=W_{0},-\kappa_{f} \frac{\partial T}{\partial z}=h_{f}\left(T_{f}-T\right), C=C_{w}, \psi=\psi_{w}, \text { at } z=0 \\
u \rightarrow 0, T \rightarrow \infty, C \rightarrow \infty, \psi \rightarrow \infty, \text { as } z \rightarrow \infty,
\end{gathered}
$$

where $\kappa_{f}=\kappa_{0} \sqrt{(1-\lambda t)}$ is the thermal conductivity of base fluid and $\kappa_{0}$ is a constant. If the Biot number, $B i=h_{f} \sqrt{v} / \kappa_{0} \sqrt{c_{0} r^{m-1}}$, is a constant and the heat transfer coefficient $h_{f}$ is proportional to $r^{(m-1) / 2}$, then the coefficient of heat transfer is expressed as $h_{f}=b_{0} r^{(m-1) / 2}$, where $b_{0}$ is constant. The Biot number can be written as $B i=h_{0} \sqrt{\left(v / c_{0}\right)} / \kappa_{0}$.

Followings are the similarity transformations to solve Equations (1)-(5) stated as (see [12,27]):

$$
\begin{gathered}
\xi=\sqrt{\frac{c_{0}}{v(1-\lambda t)} r^{(m-1) / 2} z, u}=\frac{c_{0} r^{m}}{(1-\lambda t)} \frac{d f}{d \xi}, w=-\frac{c_{0} r^{(m-1) / 2}}{(1-\lambda t)} \sqrt{\frac{v}{c_{0}}}\left(\left(\frac{m+3}{2}\right) f(\xi)+\left(\frac{m-1}{2}\right) \xi \frac{d f}{d \xi}\right), \\
\theta(\xi)=\frac{T-T_{\infty}}{T_{w}-T_{\infty}}, S(\xi)=\frac{C-C_{\infty}}{C_{w}-C_{\infty}}, \phi(\xi)=\frac{\psi-\psi_{\infty}}{\psi_{w}-\psi_{\infty}}
\end{gathered}
$$




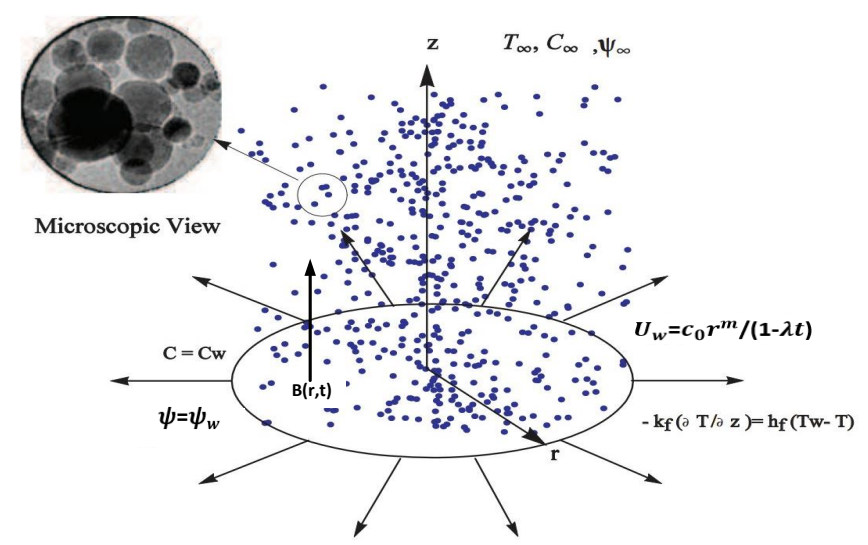

Figure 1. Physical configuration and coordinate system.

The fluid's viscosity in Equation (2) depends on the temperature and it varies exponentially. The mathematical form of the Reynolds exponential viscosity model is [29]:

$$
\mu(T)=\mu_{0} e^{-H\left(T-T_{\infty}\right)}
$$

where the dependence of intensity between $T$ and $\mu(T)$ is indicated by $\mathrm{H} . \mu_{0}$ displays the fluid's viscosity at $T_{\infty}$. Using the transformation of similarity in Equation (8) and then the Maclaurin's expansion, we get [29]:

$$
e^{-\lambda \theta}=1-\lambda \theta+O\left(\lambda^{2}\right)
$$

The following dimensionless system is now accomplished using similarity transformations (Equation (8)),

$$
\begin{aligned}
(1-\lambda \theta) \frac{d^{3} f}{d \xi^{3}}-\lambda \frac{d \theta}{d \xi} \frac{d^{2} f}{d \xi^{2}}+\left(\frac{m+3}{2}\right) f \frac{d^{2} f}{d \xi^{2}}-H a \frac{d f}{d \xi}-\sigma\left(\frac{d f}{d \xi}+\frac{1}{2} \xi \frac{d^{2} f}{d \xi^{2}}\right)-m \frac{d f^{2}}{d \xi}=0 \\
\frac{1}{P r}(1+N r) \frac{d^{2} \theta}{d \xi^{2}}-\frac{\sigma}{2} \xi \frac{d \theta}{d \xi}+\left(\frac{m+3}{2}\right) f \frac{d \theta}{d \xi}+N t\left(\frac{d \theta}{d \xi}\right)^{2}+N b \frac{d \phi}{d \xi} \frac{d \theta}{d \xi}+D f \frac{d^{2} S}{d \xi^{2}}=0 \\
\frac{1}{S c} \frac{d^{2} S}{d \xi^{2}}-\frac{\sigma}{2} \xi \frac{d S}{d \xi}+\left(\frac{m+3}{2}\right) f \frac{d S}{d \xi}+S r \frac{d^{2} \theta}{d \xi^{2}}=0 \\
\frac{d^{2} \phi}{d \xi^{2}}-\frac{\sigma L e}{2} \xi \frac{d \phi}{d \xi}+\frac{N t}{N b} \frac{d^{2} \theta}{d \xi^{2}}+L e\left(\frac{m+3}{2}\right) f \frac{d \phi}{d \xi}=0
\end{aligned}
$$

and the transformed boundary conditions in Equations (6) and (7) are:

$$
\begin{gathered}
f(\xi)=S, \frac{d f}{d \xi}=1,-\frac{d \theta}{d \xi}=-B i[1-\theta(\xi)], S(\xi)=1, \phi(\xi)=1, \quad \text { at } \quad \xi=0, \\
\frac{d f(\xi)}{d \xi} \rightarrow 0, \quad \theta(\infty) \rightarrow 0, \quad S(\infty) \rightarrow 0, \quad \phi(\infty) \rightarrow 0 \quad \text { at } \quad \xi=\infty
\end{gathered}
$$

The emerging parameters in Equations (11)-(15) are described as:

$H a=\frac{\sigma B_{0}^{2}}{\rho c_{o}}, \sigma=\frac{\lambda}{c_{0} r^{m-1}}, P r=\frac{v}{\alpha}, N b=\frac{\tau D_{B}\left(\psi_{w}-\psi_{\infty}\right)}{v}, N t=\frac{\tau D_{T}\left(T_{f}-T_{\infty}\right)}{v T_{\infty}}, D_{f}=\frac{D_{T_{c}}\left(C_{w}-C_{\infty}\right)}{v\left(T_{f}-T_{\infty}\right)}$, $S r=\frac{D_{C T}\left(T_{f}-T_{\infty}\right)}{v\left(C_{w}-C_{\infty}\right)}, S c=\frac{v}{D_{s}}, L e=\frac{\sigma}{D_{B}}, B i=h_{0} \sqrt{\left(v / c_{0}\right)} / \kappa_{0}, \lambda=H\left(T_{f}-T_{\infty}\right), S=-\frac{2 W_{0}(1-\lambda t)}{(m+3) r^{(m-1) / 2} \sqrt{c_{0} v}}$, where $H a$ is magnetic parameter, $\sigma$ is the unsteadiness, Prandtl number is Pr, Brownian motion is $N b, N t$ is the thermophoresis parameter, $D_{f}$ is the Dufour parameter, $S r$ is the Soret parameter, $S c$ is the Schmidt number, $L e$ is the Lewis number, $B i$ is the Biot number, $\lambda$ is the variable viscosity, and $S$ represents the mass transfer rate at the surface. $S>0$ indicates the suction case and $S<0$ the injection case. 


\section{Finite Element Method Solutions}

Equations (11)-(14) were solved numerically using the finite element method (FEM) under the boundary conditions in Equations (15) and (16). FEM has been used to research various computational fluid dynamics problems and is a highly efficient approach to solve various nonlinear problems [30-32]. Reddy [33] described the method of variational finite elements in excellent detail. This approach is better suited and more accurate than other numerical methods such as ADM, HPM, and FDM. It is also very proficient and has been applied in many other fields [34] to research various problems in fluid mechanics and computational fluid dynamics, solid mechanics, mass transfer, and heat transfer $[24,35,36]$. To apply FEM to the simultaneous nonlinear differential equations (Equations (11)-(14)), and to use the boundary conditions in Equations (15) and (16), we consider:

$$
\frac{d f}{d \xi}=h
$$

Equations (11)-(14) thus reduce to

$$
\begin{gathered}
(1-\lambda \theta) \frac{d^{2} h}{d \xi^{2}}-\lambda \frac{d \theta}{d \xi} \frac{d h}{d \xi}+\left(\frac{m+3}{2}\right) f \frac{d h}{d \xi}-H a h-\sigma\left(h+\frac{1}{2} \xi \frac{d h}{d \xi}\right)-m h^{2}=0, \\
\frac{1}{P r}(1+N r) \frac{d^{2} \theta}{d \xi^{2}}-\frac{\sigma}{2} \xi \frac{d \theta}{d \xi}+\left(\frac{m+3}{2}\right) f \frac{d \theta}{d \xi}+N b \frac{d \phi}{d \xi} \frac{d \theta}{d \xi}+N t\left(\frac{d \theta}{d \xi}\right)^{2}+D_{f} \frac{d^{2} S}{d \xi^{2}}=0, \\
\frac{1}{S c} \frac{d^{2} S}{d \xi^{2}}-\frac{\sigma}{2} \xi \frac{d S}{d \xi}+\left(\frac{m+3}{2}\right) f \frac{d S}{d \xi}+S r \frac{d^{2} \theta}{d \xi^{2}}=0 \\
\frac{d^{2} \phi}{d \xi^{2}}-L e \frac{\sigma}{2} \xi \frac{d \phi}{d \xi}+L e\left(\frac{m+3}{2}\right) f \frac{d \phi}{d \xi}+\frac{N t}{N b} \frac{d^{2} \theta}{d \xi^{2}}=0
\end{gathered}
$$

The corresponding boundary conditions reduce to the following form:

$$
\begin{gathered}
f(\xi)=S, h(\xi)=1,-\frac{d \theta}{d \xi}=-B i[1-\theta(\xi)], S(\xi)=1, \phi(\xi)=1, \quad \text { at } \xi=0, \\
h(\xi) \rightarrow 0, \quad \theta(\infty) \rightarrow 0, \quad S(\infty) \rightarrow 0, \quad \phi(\infty) \rightarrow 0 \quad \text { at } \quad \xi=\infty
\end{gathered}
$$

\subsection{Variational Formulations}

The weak form connected with Equations (17)-(21) over a linear element $\Omega_{a}=\left(\xi_{a}, \xi_{a+1}\right)$ is given by the following:

$$
\begin{gathered}
\int_{\xi_{a}}^{\xi_{a+1}} s_{1}\left\{\frac{d f}{d \xi}-h\right\} d \eta=0 \\
\int_{\xi_{a}}^{\xi_{a+1}} s_{2}\left\{(1-\lambda \theta) \frac{d^{2} h}{d \xi^{2}}-\lambda \frac{d \theta}{d \xi} \frac{d h}{d \xi}+\left(\frac{m+3}{2}\right) f \frac{d h}{d \xi}-H a h-\sigma\left(h+\frac{1}{2} \xi \frac{d h}{d \xi}\right)-m h^{2}\right\} d \xi=0 \\
\int_{\xi_{a}}^{\xi_{a+1}} s_{3}\left\{\frac{1}{P r}(1+N r) \frac{d^{2} \theta}{d \xi^{2}}-\frac{\sigma}{2} \xi \frac{d \theta}{d \xi}+\left(\frac{m+3}{2}\right) f \frac{d \theta}{d \xi}+N b \frac{d \phi}{d \tau} \frac{d \theta}{d \xi}+N t\left(\frac{d \theta}{d \xi}\right)^{2}+D_{f} \frac{d^{2} S}{d \xi^{2}}\right\} d \xi=0, \\
\int_{\xi_{a}}^{\xi_{a+1}} s_{4}\left\{\frac{1}{S c} \frac{d^{2} S}{d \xi^{2}}-\frac{\sigma}{2} \xi \frac{d S}{d \xi}+\left(\frac{m+3}{2}\right) f \frac{d S}{d \xi}+S r \frac{d^{2} \theta}{d \xi^{2}}\right\} d \xi=0 \\
\int_{\xi_{a}}^{\xi_{a+1}} s_{5}\left\{\frac{d^{2} \phi}{d \xi^{2}}-\frac{\sigma}{2} \xi \frac{d \phi}{d \xi}+L e\left(\frac{m+3}{2}\right) f \frac{d \phi}{d \xi}+\frac{N t}{N b} \frac{d^{2} \theta}{d \xi^{2}}\right\} d \xi=0
\end{gathered}
$$

where $s_{1}, s_{2}, s_{3}, s_{4}$, and $s_{5}$ are arbitrary test functions. 


\subsection{Finite Element Formulations}

The finite element model may be obtained from Equations (24)-(28) by plugging in the following finite element approximation form:

$$
\bar{f}=\sum_{n=1}^{2} \bar{f}_{n} \psi_{n}, \bar{h}=\sum_{n=1}^{2} \bar{h}_{n} \psi_{n}, \frac{\bar{d} \theta}{d \tilde{\zeta}}=\sum_{n=1}^{2} \frac{\bar{d} \theta_{n}}{d \tau} \psi_{n}, \frac{\bar{d} \phi}{d \bar{\zeta}}=\sum_{n=1}^{2} \frac{\bar{d} \phi_{n}}{d \xi} \psi_{n}
$$

with $s_{1}=s_{2}=s_{3}=s_{4}=s_{5}=\psi_{n}(n=1,2)$, where the test functions $\psi_{n}$ for a typical length element $\Omega_{e}=\left(\xi_{a}, \xi_{a+1}\right)$ are given by.

$$
\psi_{1}=\frac{\xi_{a+1}-\xi}{\xi_{a+1}-\xi_{a}}, \psi_{2}=\frac{\xi-\xi_{a}}{\xi_{a+1}-\xi_{a}}, \xi_{a} \leq \xi \leq \xi_{a+1} .
$$

The finite element model equations are, therefore, given by.

$$
\left[\begin{array}{lllll}
{\left[A^{11}\right]} & {\left[A^{12}\right]} & {\left[A^{13}\right]} & {\left[A^{14}\right]} & {\left[A^{15}\right]} \\
{\left[A^{21}\right]} & {\left[A^{22}\right]} & {\left[A^{23}\right]} & {\left[A^{24}\right]} & {\left[A^{25}\right]} \\
{\left[A^{31}\right]} & {\left[A^{32}\right]} & {\left[A^{33}\right]} & {\left[A^{34}\right]} & {\left[A^{35}\right]} \\
{\left[A^{41}\right]} & {\left[A^{42}\right]} & {\left[A^{43}\right]} & {\left[A^{44}\right]} & {\left[A^{45}\right]} \\
{\left[A^{51}\right]} & {\left[A^{52}\right]} & {\left[A^{53}\right]} & {\left[A^{54}\right]} & {\left[A^{55}\right]}
\end{array}\right]\left[\begin{array}{l}
\{f\} \\
\{h\} \\
\{\theta\} \\
\{S\} \\
\{\phi\}
\end{array}\right]=\left[\begin{array}{l}
\left\{b_{1}\right\} \\
\left\{b_{2}\right\} \\
\left\{b_{3}\right\} \\
\left\{b_{4}\right\} \\
\left\{b_{5}\right\}
\end{array}\right]
$$

where $A_{m n}$ are the matrices of order $2 \times 2$ and $b_{m}$ are the matrices of $2 \times 1 .(m, n=1,2,3,4,5)$

$$
\begin{aligned}
& A_{i j}^{11}=\int_{\xi_{a}}^{\xi_{a+1}} \psi_{i} \frac{d \psi_{j}}{d \tilde{\zeta}} d \xi_{,} A_{i j}^{12}=-\int_{\xi_{a}}^{\xi_{a+1}} \psi_{i} \psi_{j} d \xi_{,}, A_{i j}^{13}=A_{i j}^{14}=0, A_{i j}^{15}=A_{i j}^{21}=0, \\
& A_{i j}^{22}=-\int_{\tilde{\xi}_{a}}^{\tilde{\xi}_{a+1}} \frac{d \psi_{i}}{d \xi} \frac{d \psi_{j}}{d \xi} d \xi+\left(\frac{m+3}{2}\right) \int_{\tilde{\zeta}_{a}}^{\xi_{a+1}} \bar{f} \psi_{i} \frac{d \psi_{j}}{d \xi} d \xi-m \int_{\tilde{\xi}_{a}}^{\tilde{\xi}_{a+1}} \bar{h} \psi_{i} \psi_{j} d \xi-H a \int_{\tilde{\xi}_{a}}^{\tilde{\xi}_{a+1}} \psi_{i} \psi_{j} d \xi \\
& +\lambda \int_{\xi_{a}}^{\xi_{a+1}} \bar{\theta} \frac{d \psi_{i}}{d \xi} \frac{d \psi_{j}}{d \xi} d \xi-\lambda \int_{\xi_{a}}^{\xi_{a+1}} \bar{\phi}^{\prime} \psi_{i} \frac{d \psi_{j}}{d \xi} d \xi-\sigma \int_{\xi_{a}}^{\xi_{a+1}} \psi_{i} \psi_{j} d \xi-\frac{\xi^{\prime}}{2} \int_{\tilde{\xi}_{a}}^{\xi_{a+1}} \psi_{i} \frac{d \psi_{j}}{d \xi} d \xi, \\
& A_{i j}^{23}=0, A_{i j}^{24}=0, A_{i j}^{25}=0, A_{i j}^{31}=0, A_{i j}^{32}=0, \\
& A_{i j}^{33}=-\frac{1}{P r}(1+N r) \int_{\xi_{a}}^{\xi_{a+1}} \frac{d \psi_{i}}{d \xi} \frac{d \psi_{j}}{d \xi} d \xi-\frac{\xi}{2} \int_{\xi_{a}}^{\xi_{a+1}} \psi_{i} \frac{d \psi_{j}}{d \xi} d \xi+\left(\frac{m+3}{2}\right) \int_{\xi_{a}}^{\xi_{a+1}} \bar{f} \psi_{i} \frac{d \psi_{j}}{d \xi} d \xi, \\
& +N b \int_{\tilde{\zeta}_{a}}^{\xi_{a+1}} \bar{\phi}^{\prime} \psi_{i} \frac{d \psi_{j}}{d \xi} d \xi+N t \int_{\tilde{\zeta}_{a}}^{\xi_{a+1}} \bar{\theta}^{\prime} \psi_{i} \frac{d \psi_{j}}{d \xi} d \xi_{,}, A_{i j}^{34}=-D_{f} \int_{\tilde{\zeta}_{a}}^{\xi_{a+1}} \frac{d \psi_{i}}{d \xi} \frac{d \psi_{j}}{d \xi} d \xi, \\
& A_{i j}^{35}=0, A_{i j}^{41}=A_{i j}^{42}=0, A_{i j}^{43}=-S r \int_{\tilde{\zeta}_{a}}^{\xi_{a+1}} \frac{d \psi_{i}}{d \xi} \frac{d \psi_{j}}{d \xi} d \xi, A_{i j}^{45}=A_{i j}^{51}=A_{i j}^{52}=A_{i j}^{54}=0 \\
& A_{i j}^{44}=-\frac{1}{S c} \int_{\tilde{\zeta}_{a}}^{\tilde{\zeta}_{a+1}} \frac{d \psi_{i}}{d \xi} \frac{d \psi_{j}}{d \xi} d \xi-\frac{\xi}{2} \int_{\tilde{\zeta}_{a}}^{\tilde{\zeta}_{a+1}} \psi_{i} \frac{d \psi_{j}}{d \xi} d \xi+\left(\frac{m+3}{2}\right) \int_{\xi_{a}}^{\tilde{s}_{a+1}} \bar{f} \psi_{i} \frac{d \psi_{j}}{d \xi} d \xi_{,}, A_{i j}^{53}=-\frac{N t}{N b} \int_{\tilde{\zeta}_{a}}^{\tilde{s}_{a+1}} \frac{d \psi_{i}}{d \xi} \frac{d \psi_{j}}{d \xi} d \xi \\
& A_{i j}^{55}=-\int_{\tilde{\xi}_{a}}^{\xi_{a+1}} \frac{d \psi_{i}}{d \xi} \frac{d \psi_{j}}{d \xi} d \xi+L e\left(\frac{m+3}{2}\right) \int_{\xi_{a}}^{\xi_{a+1}} \bar{f} \psi_{i} \frac{d \psi_{j}}{d \xi} d \xi-L e \frac{\xi}{2} \int_{\xi_{a}}^{\xi_{a+1}} \psi_{i} \frac{d \psi_{j}}{d \xi} d \xi_{,}
\end{aligned}
$$


and

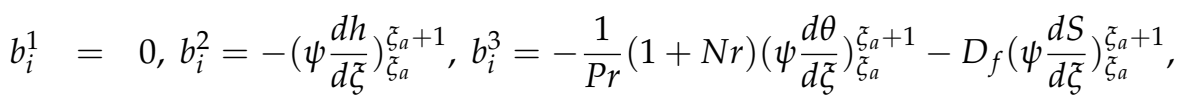

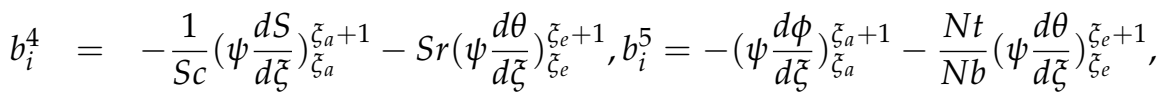

where $\bar{f}=\sum_{j=1}^{2} \bar{f}_{j} \psi_{j}, \bar{h}=\sum_{j=1}^{2} \bar{h}_{j} \psi_{j}, \frac{\bar{d} \theta}{d \bar{\zeta}}=\sum_{n=1}^{2} \frac{\bar{d} \theta_{n}}{d \bar{\zeta}} \psi_{n}$ and $\frac{\bar{d} \phi}{d \bar{\zeta}}=\sum_{n=1}^{2} \frac{\bar{d} \phi_{n}}{d \bar{\zeta}} \psi_{n}$ are supposed to be known. Equation (32) is of the order $10 \times 10$ and the whole flow domain is divided into 1000 linear elements of same size. We obtain a matrix after assembling all of the element equations. After the assemblage of the system of element equations, an ensuing system of nonlinear equations is attained. Subsequently, an iterative method is imposed to compute it for a dexterous solution. To compute $\bar{f}$, $\bar{h}, \bar{\theta}^{\prime}$, and $\bar{\phi}^{\prime}$ functions are presumed to be notorious at a lower iteration level to linearize the system. Then, the estimations for the velocity, solutal, nano-fluid, temperature, and volume fraction profile are fulfilled for a high level, and continued until the desired accuracy of 0.00005 is attained. To ensure the mesh independence, the mesh impact ability is executed. No considerable disparity in the consequences is observed for $\xi>10$. Thus, $\xi$ is fixed at 10 . For the corroboration of the convergence of consequences, we intended that the quantity of elements is enlarging $(n=100,200,400,600,700,1000,1200$, and 1300); the results are demarcated in Table 1. In addition, it was found that, as $n$ enhances further than 1200 , no considerable alteration in velocity, temperature, and concentration functions is exposed, thus the results at the final outcomes are reported for $n=1200$ elements.

Table 1. FEM Convergence results for active control of nanoparticles when $\mathrm{Pr}=0.773 ; \mathrm{Ha}=2 ; \mathrm{Nb}=$ $S=N t=0.3, S c=5, D_{f}=S r=0.1 ; L e=2 ; m=2, B i=1, \lambda=N r=0.5$, and $\sigma=0.2$.

\begin{tabular}{cccccc}
\hline Number of Elements & $\boldsymbol{f ( 1 . 3 )}$ & $\boldsymbol{h ( 1 . 3 )}$ & $\boldsymbol{\theta ( 1 . 3 )}$ & $\boldsymbol{S ( \mathbf { 1 . 3 } )}$ & $\boldsymbol{\phi ( 1 . 3 )}$ \\
\hline 100 & 0.643350 & 0.031597 & 0.019678 & 0.389363 & 0.130666 \\
200 & 0.644363 & 0.031894 & 0.019659 & 0.388431 & 0.130627 \\
400 & 0.644616 & 0.031968 & 0.019656 & 0.388199 & 0.130617 \\
600 & 0.644663 & 0.031982 & 0.019655 & 0.388156 & 0.130616 \\
700 & 0.644673 & 0.031984 & 0.019655 & 0.388147 & 0.130615 \\
1000 & 0.644687 & 0.031989 & 0.019654 & 0.388134 & 0.130615 \\
1200 & 0.644691 & 0.031989 & 0.019654 & 0.388130 & 0.130615 \\
1300 & 0.644692 & 0.031990 & 0.019654 & 0.388130 & 0.130615 \\
\hline
\end{tabular}

\section{Results and Discussion}

To determine the unknown functions of velocity profile, temperature, and concentration profiles, the above set of Equations (11)-(14) and boundary conditions in Equations (15) and (16) were solved using the variational finite element method. We validated our results with the existing literature listed in Tables 2 and 3 before plotting the results.

Table 2. The coefficient of skin friction comparison with different values of magnetic parameter M.

\begin{tabular}{ccccc}
\hline$S$ & $\sigma$ & $\begin{array}{c}\text { Azeem et al. [12] } \\
\text { (HAM) }\end{array}$ & $\begin{array}{c}\text { Faraz et al. [22] } \\
\text { (KBM) }\end{array}$ & $\begin{array}{c}\text { (Our Results) } \\
\text { (FEM) }\end{array}$ \\
\hline-1.0 & 0.5 & 0.620400 & 0.620436 & 0.620437 \\
-0.5 & & 0.887200 & 0.887247 & 0.887247 \\
0.0 & & 1.308999 & 1.308670 & 1.308668 \\
0.5 & & 1.907999 & 1.907973 & 1.907973 \\
1.0 & & 2.655999 & 2.655591 & 2.655588 \\
0.5 & 0.0 & 1.798999 & 1.798668 & 1.798667 \\
& 0.5 & 1.907999 & 1.907973 & 1.907972 \\
& 1.0 & 2.016999 & 2.016665 & 2.016667 \\
\hline
\end{tabular}


Table 3. Values of Reduced Nusselt and Sherwood Numbers Nur and Shr for Different Values of $\mathrm{Pr}$, $L e$, and $B i$ when $N b=N t=0.1, m=1$, and other parameters are zero.

\begin{tabular}{|c|c|c|c|c|c|c|}
\hline \multirow{2}{*}{$B i$} & \multirow{2}{*}{ Le } & \multirow{2}{*}{$\operatorname{Pr}$} & \multicolumn{2}{|c|}{ Mustafa et al. [4] } & \multicolumn{2}{|c|}{ FEM (Present Results) } \\
\hline & & & $-\theta^{\prime}(0)$ & $-\phi^{\prime}(0)$ & $-\theta^{\prime}(0)$ & $-\phi^{\prime}(0)$ \\
\hline 0.1 & 1.0 & 1.00 & 0.088980 & 0.793545 & 0.088980 & 0.793541 \\
\hline 0.4 & & & 0.266901 & 0.676928 & 0.266900 & 0.676926 \\
\hline 1.0 & & & 0.443452 & 0.561546 & 0.443450 & 0.561546 \\
\hline 2.0 & & & 0.567859 & 0.480446 & 0.567857 & 0.480448 \\
\hline \multirow[t]{4}{*}{0.5} & 0.4 & 1.00 & 0.310557 & 0.193614 & 0.310540 & 0.195635 \\
\hline & 0.7 & & 0.308900 & 0.446367 & 0.308899 & 0.446420 \\
\hline & 2.0 & & 0.305905 & 1.160300 & 0.305904 & 1.160294 \\
\hline & 10.0 & & 0.303021 & 3.222890 & 0.303021 & 3.222894 \\
\hline \multirow[t]{4}{*}{0.5} & 1.00 & 0.40 & 0.230078 & 0.710803 & 0.230424 & 0.711456 \\
\hline & & 0.72 & 0.281948 & 0.671917 & 0.281951 & 0.671938 \\
\hline & & 2.00 & 0.353934 & 0.605337 & 0.353933 & 0.605334 \\
\hline & & 10.0 & 0.422650 & 0.515390 & 0.422649 & 0.515386 \\
\hline
\end{tabular}

We found an excellent agreement between our solution and that of the already published research articles; this confirms the validity and accuracy of the present results. Variation of existing parameters $\mathrm{Ha}, \mathrm{m}, \sigma, \operatorname{Pr}, \mathrm{Nb}, \mathrm{Nt}, \mathrm{D}_{f}, \mathrm{Sr}, \mathrm{Sc}, \mathrm{Le}, \mathrm{Bi}, \mathrm{S}$, and $\lambda$ are plotted in Figure 2. Profile functions for velocity, temperature, and concentration, for different value of viscosity parameter, magnitude of velocity, temperature, and concentration profiles are given in all upcoming figures: (i) $\lambda=0$ (shown as green solid lines); and (ii) $\lambda=0.5$ (shown as red dotted lines). The base amount of these parameters are considered to be $\mathrm{Pr}=0.773, \mathrm{Ha}=2.0, \mathrm{Nb}=\mathrm{N} t=S=0.3, \mathrm{Sc}=5.0, \mathrm{D}_{f}=S r=0.1, L e=m=2.0$, $B i=1.0, R_{d}=0.3, N r=0.5$, and $\sigma=0.2$.
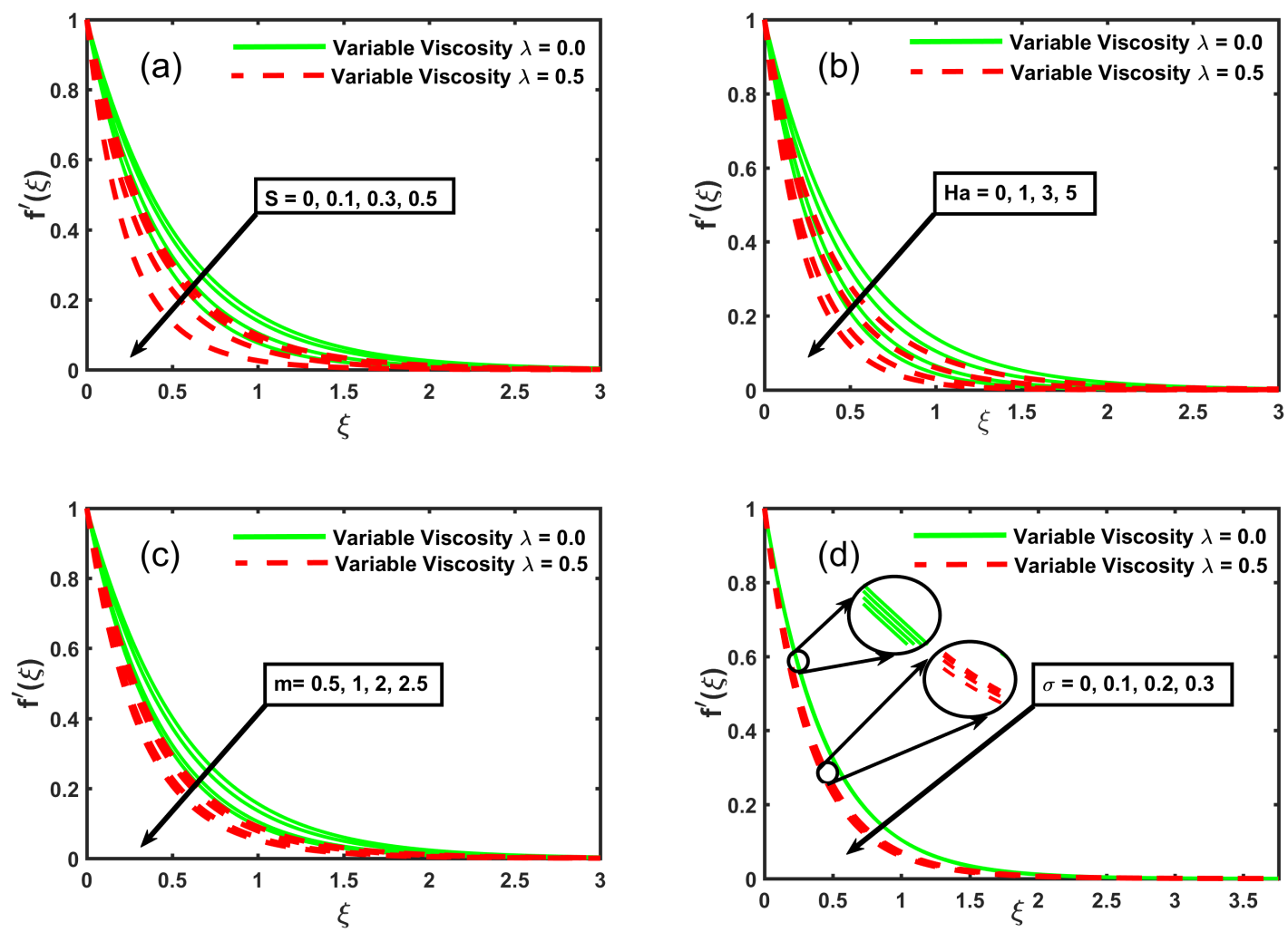

Figure 2. Impact of $f^{\prime}(\xi)$ for suction $S$, magnetic field Ha, stretching sheet $m$, unsteadiness $\sigma$, and variable viscosity $\lambda$. 
Figure 2 a shows the effect of the suction $S$ and variable viscosity $\lambda$ parameters. In the velocity function, the decline is observed when the suction is $S>0$ and the fluid velocity is decreased for $\lambda>0$. Figure $2 a$ depicts that the fluid flow velocity displays a decreasing trend with the rising values of $\lambda$. The reason behind this is the rise in viscous effects with a higher viscosity, which decreases the fluid flow as a result. In addition, the thickness of the momentum boundary layer has been perceived as an enhancement. Likewise, with the impacts of the magnetic field, one can observe retardation in the velocity activity (see Figure $2 b$ ). This is because of the forces of Lorentz, which act as resistive forces and thus oppose the movement of fluids. Therefore, the magnetic field manages the fluid flow successfully. Figure $2 \mathrm{c}$ shows the variation of stretching sheet parameter $m$ on the fluid velocity. The magnitude of the velocity's radial component decreases as $m$ increases. $m$, in contrast, has a significant impact on $\xi$ and the velocity components $u$ and $w$, respectively. A similar trend is observed in variable viscosity $\lambda$. Figure $2 \mathrm{~d}$ shows that, with rising values of $\sigma>0$, the velocity profiles decrease, and a similar trend is observed for the growing value of the viscosity variable.
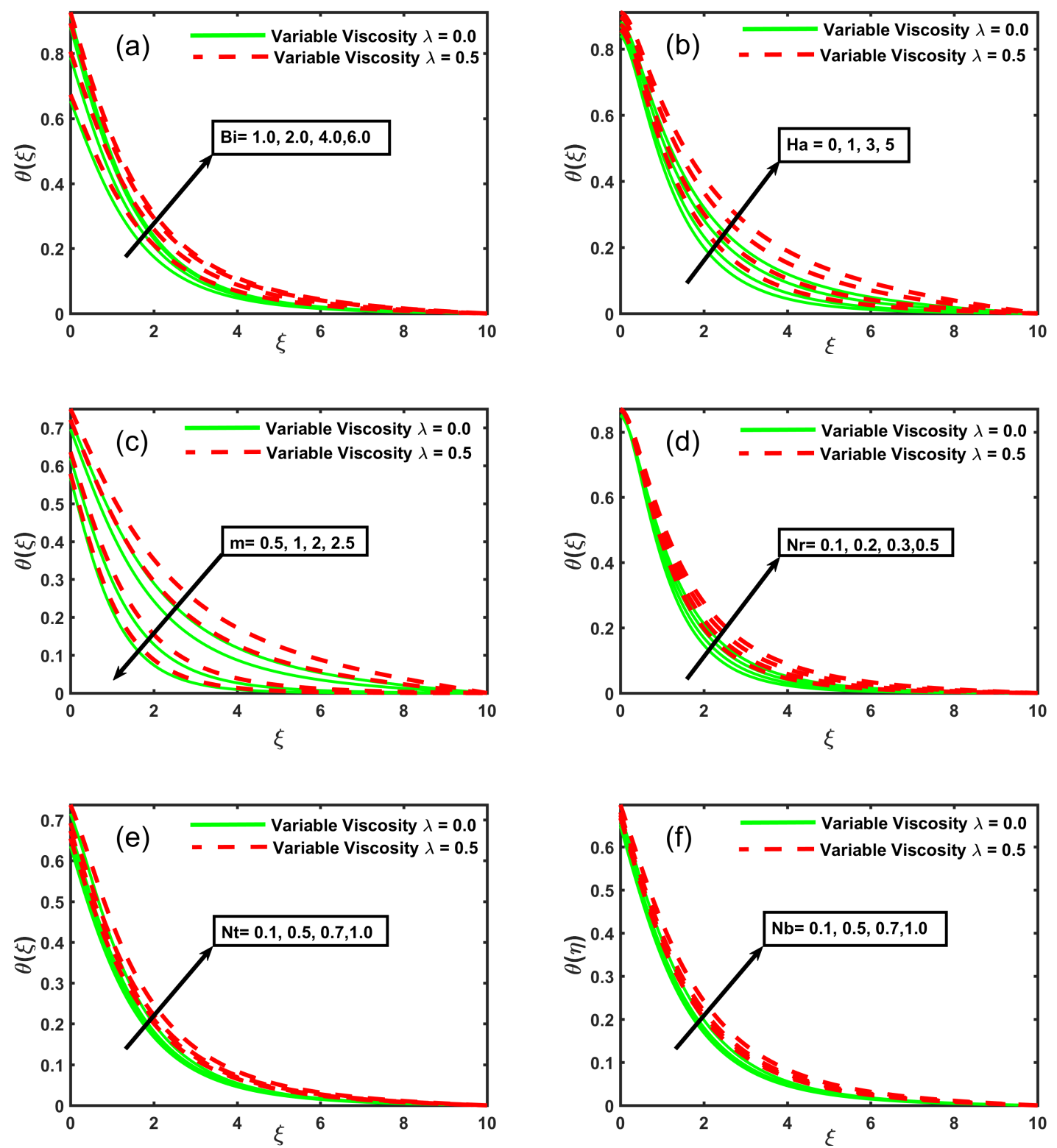

Figure 3. Impact of $\theta^{\prime}(\xi)$ for Biot number $B i$, magnetic field $\mathrm{Ha}$, stretching sheet $m$, radiation $N r$, thermophoresis $N t$, Brownian motion $N b$, and variable viscosity $\lambda$. 
The effect of varying Biot number $B i$ values on the distribution of temperature are shown in Figure $3 a$. The thermal layer shows an increase with the $B i$ increasing values caused by the greater temperature of the fluid. Figure $3 \mathrm{~b}$ illustrates the effect of the Hartmann number on the temperature distribution. As the value of Hartmann's number increases, the temperature rises. The increasing magnetic field increases the friction impacts, which heats up the fluid and thus increases the fluid's temperature. In contrast, with the rise in variable viscosity $\lambda$, the thickness of the thermal boundary layer also increases. The temperature profile decreases as the value of $m$ increases. The thermal boundary layer thickness is often shown to be greater than $m$ when considering the parameter viscosity $\lambda=0.5$ (see Figure $3 \mathrm{c}$ ). Figure $3 \mathrm{~d}$ displays temperature trends for different viscosity parameter $\lambda$ and radiation parameter $N r$ values. With the rising viscosity parameter $\lambda$, the temperature rises. Moreover, the thermal boundary layer thickness is also increasing. Figure $3 \mathrm{~d}$ illustrates the impact of the radiation parameter on the temperature distribution. As the value of radiation $N r$ increases, the temperature rises. Temperature behavior is shown in Figure 3e,f, respectively, under the impact of thermophoresis $\mathrm{N} t$ and Brownian motion $\mathrm{Nb}$. The temperature is decreasing with the increasing number of $\mathrm{N} t$ and the value of $\mathrm{Nb}$.

Figure $4 \mathrm{a}, \mathrm{b}$ show the effect on the dimensionless concentration profile of the stretching parameter $m$, the Schmidt number $S c$, and the viscosity parameter $\lambda$. For distinct values of $\lambda=0$ and $\lambda=0.5$, an increasing behavior is shown in the concentration profile. Moreover, the concentration profile decreases as the value of $m$ and $S c$ increases. The influences of the thermophoresis parameter $N t$, the Brownian motion parameter $N b$, and the viscosity parameter $\lambda$ on the dimensionless nanoparticle concentration profiles are sketched in Figure $4 \mathrm{c}, \mathrm{d}$. The variation in nanoparticle concentration profile because of changing $N t$ is shown in Figure 4c. The nanoparticle concentration profile demonstrates a strong decreasing behavior at and near the wall with the increasing thermophoresis parameter $\mathrm{Nt}$.
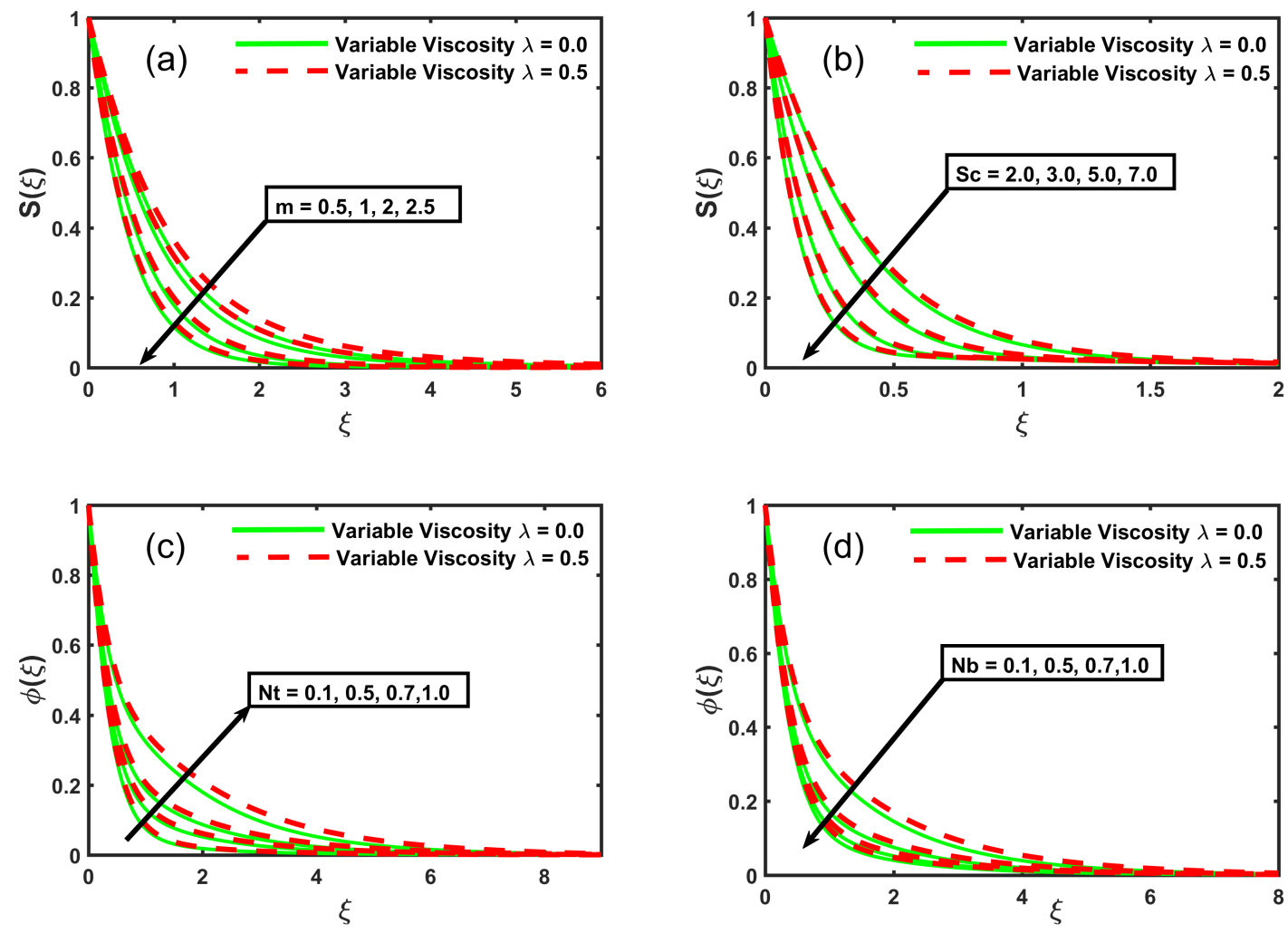

Figure 4. Impact of $S(\xi)$ and $\phi(\xi)$ for stretching sheet $m$, Schmidt number $S c$, thermophoresisbNt, Brownian motion $N b$, and variable viscosity $\lambda$. 
On the other hand, relative to the thermophoresis parameter $N t$, the effect of the Brownian motion parameter $\mathrm{Nb}$ is quite different (see Figure $4 \mathrm{~d}$ ). The nanoparticle concentration profile magnitude depicts a declining behavior with rising $\mathrm{Nb}$ values. Moreover, with the rising viscosity parameter $\lambda$, the nanoparticle concentration profile rises.

In Figure $5 \mathrm{a}$, with the increasing value of suction $S$, stretching sheet parameter $m$, and viscosity variable parameter $\lambda$, one can observe an increase in the magnitude of $C f R e_{x}^{1 / 2}$. Figure $5 \mathrm{~b}$ elaborates the variance for different $H a, \lambda$, and $N r$ values in the Nusselt number coefficient $N u R e_{x}^{-1 / 2}$. The magnitude of $N u R e_{x}^{-1 / 2}$ shows a decline on increasing values of $H a$ and $\lambda$. Moreover, the inverse behavior of $\mathrm{Nr}$ is observed. A declining trend is observed in terms of Sherwood number coefficient $S h r e_{x}^{1 / 2}$ as $H a, \lambda$, and $N t$ increase. Figure $5 c$ clearly shows the decreasing value of Sherwood number coefficient $\operatorname{ShrRe} e_{x}^{1 / 2}$.
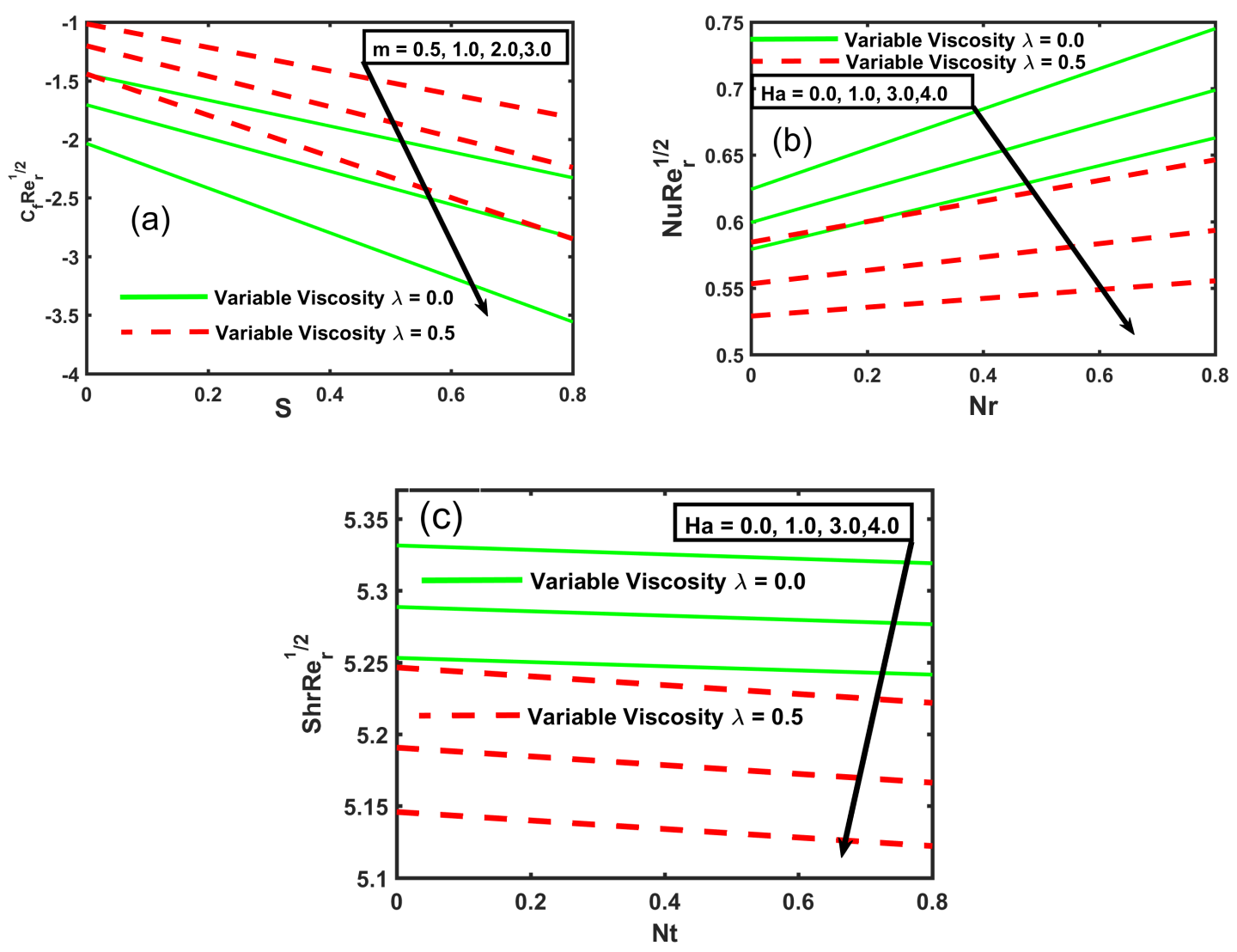

Figure 5. Impact of $C f R e_{x}^{1 / 2}, N u R e_{x}^{-1 / 2}$, and $S h r R e_{x}^{1 / 2}$ for suction $S$, stretching sheet $m$, magnetic field $\mathrm{Ha}$, radiation $N r$, thermophoresisb $N t$, and variable viscosity $\lambda$.

Table 4 is structured with the numerical results of the skin friction coefficient, Nusselt number $-\theta^{\prime}(0)$, and Sherwood number $-\phi^{\prime}(0)$ for various value of $m, H a, B i$, and $S c$ by assuming the cases of variable viscosity $\lambda=0$ and $\lambda=0.5$, respectively. In this table, an increase in the magnitude of the skin friction coefficient, Nusselt number $-\theta^{\prime}(0)$, and Sherwood number $-\phi^{\prime}(0)$ can be observed with the increasing viscosity parameter values $(\lambda), \mathrm{m}$, and Ha; however, for the magnitude of Nusselt and Sherwood numbers against Hartman number $\mathrm{Ha}$, the opposite behavior is recorded. The decline in Nusselt number and Sherwood number magnitude can be observed with the increasing viscosity parameter values $(\lambda)$ and $B i$, but the opposite behavior is recorded for the magnitude of Nusselt and Sherwood numbers against Schmidt number Sc (see Table 4). 
Table 4. Assessment of $-f^{\prime \prime}(0),-\theta^{\prime}(0)$, and $-\phi^{\prime}(0)$ for numerous values of $m, H a, B i$, and $S c$ when $N b=0.3 ; N t=0.3 ; \sigma=0.2 ; L e=2 ; D_{f}=0.1 ; S r=0.1 ; N r=0.5 ; S=0.3$; and $\operatorname{Pr}=0.773$.

\begin{tabular}{|c|c|c|c|c|c|c|c|c|c|}
\hline \multirow{2}{*}{$m$} & \multirow{2}{*}{$\mathrm{Ha}$} & \multirow{2}{*}{$B i$} & \multirow{2}{*}{$S c$} & \multicolumn{3}{|c|}{ Variable Viscosity $\lambda=0$} & \multicolumn{3}{|c|}{ Variable Viscosity $\lambda=0.5$} \\
\hline & & & & $-f^{\prime \prime}(0)$ & $-\theta^{\prime}(0)$ & $-\phi^{\prime}(0)$ & $-f^{\prime \prime}(0)$ & $-\theta^{\prime}(0)$ & $-\phi^{\prime}(0)$ \\
\hline 1.0 & 1.0 & 1.0 & 5.0 & 1.917015 & 0.197352 & 1.966102 & 1.500390 & 0.160016 & 1.887431 \\
\hline 3.0 & & & & 2.531770 & 0.249901 & 2.677550 & 2.030868 & 0.222542 & 2.586780 \\
\hline 5.0 & & & & 3.066937 & 0.282943 & 3.365334 & 2.500620 & 0.260220 & 3.268914 \\
\hline \multirow[t]{3}{*}{1.0} & 1.0 & 1.0 & 5.0 & 1.917015 & 0.197352 & 1.966102 & 1.500390 & 0.160016 & 1.887431 \\
\hline & 3.0 & & & 2.445511 & 0.161933 & 1.897593 & 1.886349 & 0.117210 & 1.812817 \\
\hline & 5.0 & & & 2.868619 & 0.137579 & 1.853265 & 2.187080 & 0.091258 & 1.768997 \\
\hline \multirow[t]{3}{*}{1.0} & 1.0 & 1.0 & 5.0 & 1.917015 & 0.197352 & 1.966102 & 1.500390 & 0.160016 & 1.887431 \\
\hline & & 2.0 & & 1.917015 & 0.234919 & 1.939081 & 1.460656 & 0.181408 & 1.858082 \\
\hline & & 4.0 & & 1.917015 & 0.259440 & 1.921454 & 1.436016 & 0.193590 & 1.840234 \\
\hline \multirow[t]{3}{*}{1.0} & 1.0 & 1.0 & 5.0 & 1.917015 & 0.197352 & 1.966102 & 1.500390 & 0.160016 & 1.887431 \\
\hline & & & 7.0 & 1.917015 & 0.148547 & 2.018283 & 1.472408 & 0.105062 & 1.932371 \\
\hline & & & 9.0 & 1.917015 & 0.100783 & 2.070145 & 1.443607 & 0.050675 & 1.976854 \\
\hline
\end{tabular}

\section{Conclusions}

This investigation comprised the numerical study of the magnetohydrodynamic (MHD) nanofluid flow over a radially stretching sheet along with the viscosity dependent on temperature, convective boundary condition, thermo-diffusion, and the radiation effects. Using the suitable similarity transformation, nonlinear PDEs are changed into a set of highly nonlinear ODEs, and nonlinear coupled ODEs were solved using a robust variational finite element (FEM) method. Moreover, graphic pictures demonstrating the effect of different emerging parameters on the properties of fluid velocity, temperature and concentration profiles are also given. The main findings of this research article are as follows:

- The velocity of fluid declines with the increasing values of suction, Hartmann number, stretching sheet, and unsteadiness parameters.

- The temperature rises with the increasing values of Hartmann number, radiation, thermophoresis, Brownian motion, and Biot number parameters.

- The solutal concentration profile illustrates a decreasing impact with the growing of the stretching sheet parameter and the Schmidt number.

- The concentration profile of nanoparticles indicates a decline with the growing values of the Brownian motion, Moreover, the elevation of the thermophoresis parameter enhances the concentration profile of nanoparticles.

- The growing values of the viscosity parameter decline the velocity of nanofluid but elevates the concentration and temperature profiles.

- An increase in the magnitude of skin friction coefficient, Nusselt number, and Sherwood number can be detected with the growing viscosity parameter values, stretching sheet parameter, and Hartmann number; however, for the magnitude of Nusselt and Sherwood numbers against Hartman number, an opposite behavior was noted.

- The decline in Nusselt number and Sherwood number magnitude was recorded with the increasing viscosity parameter values and Biot number, but an opposite behavior was recorded for the magnitude of Nusselt and Sherwood numbers against Schmidt number.

- The present study was restricted to the unsteady state flow and neglected the slip effects at the sheet. For future study, stagnation point flow with viscous dissipation effects at the sheet will be examined.

Author Contributions: B.A. and R.A.N. modeled the problem and wrote the manuscript. Y.N. thoroughly checked the mathematical modeling and made English corrections. B.A. solved the problem using MATLAB software. M.T.S., S.A.K., A.U.R. and S.A. reviewed and edited the manuscript. Y.N. contributed to the results and discussions. All authors have read and agreed to the published version of the manuscript.

Funding: This research received no external funding.

Conflicts of Interest: The authors declare no conflict of interest. 


\section{Nomenclature}

$\mathrm{Ha} \quad$ Magnetic parameter

$\mathrm{Nb} \quad$ Brownian motion parameter

Nt Thermophoresis parameter

Pr Prandtl number

Le Lewis number

$D_{f} \quad$ Dufour parameter

Sr Soret parameter

Sc Schmidt number

$S \quad$ Mass transfer rate at the surface

$\lambda \quad$ Variable viscosity

$\sigma^{*} \quad$ Electrical conductivity

$\alpha \quad$ Thermal diffusivity

T Temperature

$\sigma \quad$ unsteadiness parameter

$\beta \quad$ Biot number

$T_{w} \quad$ Sheet temperature

$T_{\infty} \quad$ Ambient temperature

$C_{w} \quad$ Solutal concentration

$\mathrm{C}_{\infty} \quad$ Ambient solutal concentration

$U_{w}(r, t) \quad$ Velocity of sheet

$\phi_{w} \quad$ Nanoparticle volume fraction

$D_{T} \quad$ Thermal diffusivity

$D_{S} \quad$ Molecular diffusivity

$D_{B} \quad$ Brownian diffusivity

$D_{C T} \quad$ Soret diffusivity

$D_{T c} \quad$ Dufour diffusivity

$\mu \quad$ Dynamic viscosity

$\rho \quad$ Fluid density

$u, w \quad$ Velocity components

$\psi_{n} \quad$ test functions

\section{References}

1. Aziz, A.; Khan, W.A.; Pop, I. Free convection boundary layer flow past a horizontal flat plate embedded in porous medium filled by nanofluid containing gyrotactic microorganisms. Int. J. Therm. Sci. 2012, 56, 48-57. [CrossRef]

2. Choi, S.. Enhancing thermal conductivity of fluids with nanoparticle, development and applications of non-newtonian flow. ASME Fluids Eng. Div. 1995, 231, 99-105.

3. Ariel, P.D. Axisymmetric flow of a second grade fluid past a stretching sheet. Int. J. Eng. Sci. 2001, 39, 529-553. [CrossRef]

4. Mustafa, M.; Hayat, T.; Alsaedi, A. Axisymmetric flow of a nanofluid over a radially stretching sheet with convective boundary conditions. Curr. Nanosci. 2012, 8, 328-334. [CrossRef]

5. Akbari, O.A.; Toghraie, D.; Karimipour, A.; Marzban, A.; Ahmadi, G.R. The effect of velocity and dimension of solid nanoparticles on heat transfer in non-Newtonian nanofluid. Phys. E Low-Dimens. Syst. Nanostruct. 2017, 86, 68-75. [CrossRef]

6. Mohyud-Din, S.T.; Khan, U.; Ahmed, N.; Rashidi, M.M. A study of heat and mass transfer on magnetohydrodynamic (MHD) flow of nanoparticles. Propuls. Power Res. 2018, 7, 72-77. [CrossRef]

7. Raza, J. Thermal radiation and slip effects on magnetohydrodynamic (MHD) stagnation point flow of Casson fluid over a convective stretching sheet. Propuls. Power Res. 2019. [CrossRef]

8. Ashraf, M.; Bashir, S. Numerical simulation of MHD stagnation point flow and heat transfer of a micropolar fluid towards a heated shrinking sheet. Int. J. Numer. Methods Fluids 2012, 69, 384-398. [CrossRef]

9. Chen, C.H. Laminar mixed convection adjacent to vertical, continuously stretching sheets. Heat Mass Transf./Waerme- und Stoffuebertragung 1998, 33, 471-476. [CrossRef] 
10. Sankara, K.K.; Watson, L.T. Micropolar flow past a stretching sheet. Z. Angew. Math. Phys. 1985, 36, 845-853. [CrossRef]

11. Hamza, E. The magnetohydrodynamic squeeze film. J. Tribol. 1988, 110, 375-377. [CrossRef]

12. Shahzad, A.; Ali, R.; Hussain, M.; Kamran, M. Unsteady axisymmetric flow and heat transfer over time-dependent radially stretching sheet. Alex. Eng. J. 2017, 56, 35-41. [CrossRef]

13. Shahzad, A.; Ahmed, J.; Khan, M. On heat transfer analysis of axisymmetric flow of viscous fluid over a nonlinear radially stretching sheet. Alex. Eng. J. 2016, 55, 2423-2429. [CrossRef]

14. Rana, P.; Bég, O.A. Mixed convection flow along an inclined permeable plate: effect of magnetic field, nanolayer conductivity and nanoparticle diameter. Appl. Nanosci. 2015, 5, 569-581. [CrossRef]

15. Thumma, T.; Anwar Bég, O.; Kadir, A. Numerical study of heat source/sink effects on dissipative magnetic nanofluid flow from a nonlinear inclined stretching/shrinking sheet. J. Mol. Liquids 2017, 232, 159-173. [CrossRef]

16. Seth, G.S.; Sarkar, S. MHD natural convection heat and mass transfer flow past a time dependent moving vertical plate with ramped temperature in a rotating medium with hall effects, radiation and chemical reaction. J. Mech. 2015, 31, 91-104. [CrossRef]

17. Takhar, H.S.; Agarwal, R.S.; Bhargava, R.; Jain, S. Mixed convection flow of a micropolar fluid over a stretching sheet. Heat Mass Transf./Waerme- und Stoffuebertragung 1998, 34, 213-219. [CrossRef]

18. Qiu, L.; Zhu, N.; Feng, Y.; Michaelides, E.E.; Żyła, G.; Jing, D.; Zhang, X.; Norris, P.M.; Markides, C.N.; Mahian, O. A review of recent advances in thermophysical properties at the nanoscale: From solid state to colloids. Phys. Rep. 2019. [CrossRef]

19. Dhanai, R.; Rana, P.; Kumar, L. Critical values in slip flow and heat transfer analysis of non-Newtonian nanofluid utilizing heat source/sink and variable magnetic field: Multiple solutions. J. Taiwan Inst. Chem. Eng. 2016, 58, 155-164. [CrossRef]

20. Nayak, B.; Mishra, S.R.; Krishna, G.G. Chemical reaction effect of an axisymmetric flow over radially stretched sheet. Propuls. Power Res. 2019, 8, 79-84. [CrossRef]

21. Ashraf, M.; Batool, K. Mhd Flow and Heat Transfer of a Micropolar Fluid Over a Stretchable Disk. J. Theor. Appl. Mech. 2013, 51, 25-38.

22. Faraz, F.; Imran, S.M.; Ali, B.; Haider, S. Thermo-diffusion and multi-slip effect on an axisymmetric Casson flow over a unsteady radially stretching sheet in the presence of chemical reaction. Processes 2019, 7, 851. [CrossRef]

23. Khan, S.A.; Nie, Y.; Ali, B. Multiple Slip Effects on Magnetohydrodynamic Axisymmetric Buoyant Nanofluid Flow above a Stretching Sheet with Radiation and Chemical Reaction. Symmetry 2019, 11, 1171. [CrossRef]

24. Uddin, M.; Rana, P.; Bég, O.A.; Ismail, A.M. Finite element simulation of magnetohydrodynamic convective nanofluid slip flow in porous media with nonlinear radiation. Alex. Eng. J. 2016, 55, 1305-1319. [CrossRef]

25. Kumar, L. Finite-element analysis of transient heat and mass transfer in microstructural boundary layer flow from a porous stretching sheet. Comput. Therm. Sci. Int. J. 2014, 6. [CrossRef]

26. Shankar, B.; Yirga, Y. Unsteady heat and mass transfer in MHD flow of nanofluids over stretching sheet with a non-uniform heat source/sink. Int. J. Math. Comput. Stat. Nat. Phys. Eng. 2013, 7, 1248-1255.

27. Sajid, M.; Hayat, T.; Asghar, S.; Vajravelu, K. Analytic solution for axisymmetric flow over a nonlinearly stretching sheet. Arch. Appl. Mech. 2008, 78, 127-134. [CrossRef]

28. Khan, M.; Malik, R.; Munir, A.; Shahzad, A. MHD flow and heat transfer of Sisko fluid over a radially stretching sheet with convective boundary conditions. J. Braz. Soc. Mech. Sci. Eng. 2016, 38, 1279-1289. [CrossRef]

29. Disu, A.; Dada, M. Reynold's model viscosity on radiative MHD flow in a porous medium between two vertical wavy walls. J. Taibah Univ. Sci. 2017, 11, 548-565. [CrossRef]

30. Gupta, D.; Kumar, L.; Bég, O.A.; Singh, B. Finite element analysis of MHD flow of micropolar fluid over a shrinking sheet with a convective surface boundary condition. J. Eng. Thermophys. 2018, 27, 202-220. [CrossRef]

31. Bhargava, R.; Rana, P. Finite element solution to mixed convection in MHD flow of micropolar fluid along a moving vertical cylinder with variable conductivity. Int. J. Appl. Math. Mech. 2011, 7, $29-51$.

32. Rana, P.; Bhargava, R.; Bég, O.A. Finite element simulation of unsteady magneto-hydrodynamic transport phenomena on a stretching sheet in a rotating nanofluid. Proc. Inst. Mech. Eng. Part N J. Nanoeng. Nanosyst. 2013, 227, 77-99. [CrossRef] 
33. Reddy, J.N. Solutions Manual for an Introduction to the Finite Element Method; McGraw-Hill: New York, NY, USA, 1993; p. 41.

34. Ali, L.; Liu, X.; Ali, B.; Mujeed, S.; Abdal, S. Finite Element Simulation of Multi-Slip Effects on Unsteady MHD Bioconvective Micropolar nanofluid Flow Over a Sheet with Solutal and Thermal Convective Boundary Conditions. Coatings 2019, 9, 842. [CrossRef]

35. Ali, L.; Liu, X.; Ali, B.; Mujeed, S.; Abdal, S. Finite Element Analysis of Thermo-Diffusion and Multi-Slip Effects on MHD Unsteady Flow of Casson Nano-Fluid over a Shrinking/Stretching Sheet with Radiation and Heat Source. Appl. Sci. 2019, 9, 5217. [CrossRef]

36. Ibrahim, W.; Gadisa, G. Finite Element Method Solution of Boundary Layer Flow of Powell-Eyring Nanofluid over a Nonlinear Stretching Surface. J. Appl. Math. 2019, 2019. [CrossRef]

(C) 2020 by the authors. Licensee MDPI, Basel, Switzerland. This article is an open access article distributed under the terms and conditions of the Creative Commons Attribution (CC BY) license (http:// creativecommons.org/licenses/by/4.0/). 